\title{
Comparison of HbA1c and FPG for the diagnosis of type 2 diabetes- A population based study from urban and rural Delhi, India
}

\author{
Pradeep A Praveen', Kalpana Singh'2,3, Ritvik Amarchand1, Ruby Gupta ${ }^{3}$, Lakshmy Ramakrishnan ${ }^{1}$, Dimple \\ Kondal $^{3}$, K Srinath Reddy ${ }^{3}$, Ambuj Roy', Anand Krishnan', Dorairaj Prabhakaran,3, Nikhil Tandon ${ }^{1}$
}

\section{${ }^{1}$ All India Institute of Medical Sciences, New Delhi, India, ${ }^{2}$ Centre for Chronic Disease Control, New Delhi, India, ${ }^{3}$ Public Health Foundation of India, Gurugram, India}

\section{Background}

Glycated Hemoglobin ( $\mathrm{HbA} 1 \mathrm{c})$ has been introduced as a complementary diagnostic test for type 2 diabetes and prediabetes. However, its performance as a screening test has not been well characterized in many ethnic groups including Asian Indians.

\section{Aim}

This study evaluated the concordance between $\mathrm{HbA1c}$ and Fasting Plasma Glucose (FPG) in the diagnosis of prediabetes and diabetes in Indian population.

\section{Methods}

* A representative cross-sectional survey was carried out in urban and rural areas of the National Capital Region of Delhi, India among adults aged 30 years and above using a multistage cluster random sampling method.

* Individuals with medical history of diabetes and those who have not provided blood samples were excluded from data analysis.

* An individual was diagnosed to have diabetes, if the $\mathrm{HbA} 1 \mathrm{c}$ value was $\geq 6.5 \%$. Pre-diabetes was defined as $\mathrm{HbA1c}$ value between $5.7 \%$ and $6.4 \%$.

* In addition and for comparison, diabetes was also diagnosed as individuals with FPG $\geq 126 \mathrm{mg} / \mathrm{dl}$, and prediabetes was classified as FPG between $100 \mathrm{mg} / \mathrm{dl}$ and $125 \mathrm{mg} / \mathrm{dl}$.

\section{Results}

* The study included 3878 individuals (1729 males and 2149 women) with mean (SD) age of $47.2(13.5)$ years.

* Based on HbA1c, the prevalence of newly diagnosed diabetes and pre-diabetes was $6.1 \%$ [95\%Cl: $5.4 \%-$ $6.9 \%$ ] and $16.9 \%$ [95\%Cl: 15.8\%-18.1\%] respectively.

* Using FPG based criteria, the prevalence of diabetes and pre-diabetes was $7.2 \%$ [95\%Cl: $6.4 \%-8.0 \%]$ and $38.7 \%$ [95\%Cl: 37.1\%-40.3\%].

* There is significant discordance in the diagnosis of diabetes and pre-diabetes between FPG and HbA1c measurements (Table 1).

* Those with isolated high HbA1c were significantly inactive, overweight/obese, hypertensive with unfavourable total cholesterol compared to isolated high FPG group (Table 2).

* Those who were categorised as 'diabetes' by both FPG and HbA1c criteria were predominantly males with poor cardiovascular risk compared to isolated high FPG, isolated high $\mathrm{HbA} 1 \mathrm{c}$ and normoglycemic groups.
Table-1: Diagnostic classifications by HbA1c and FPG

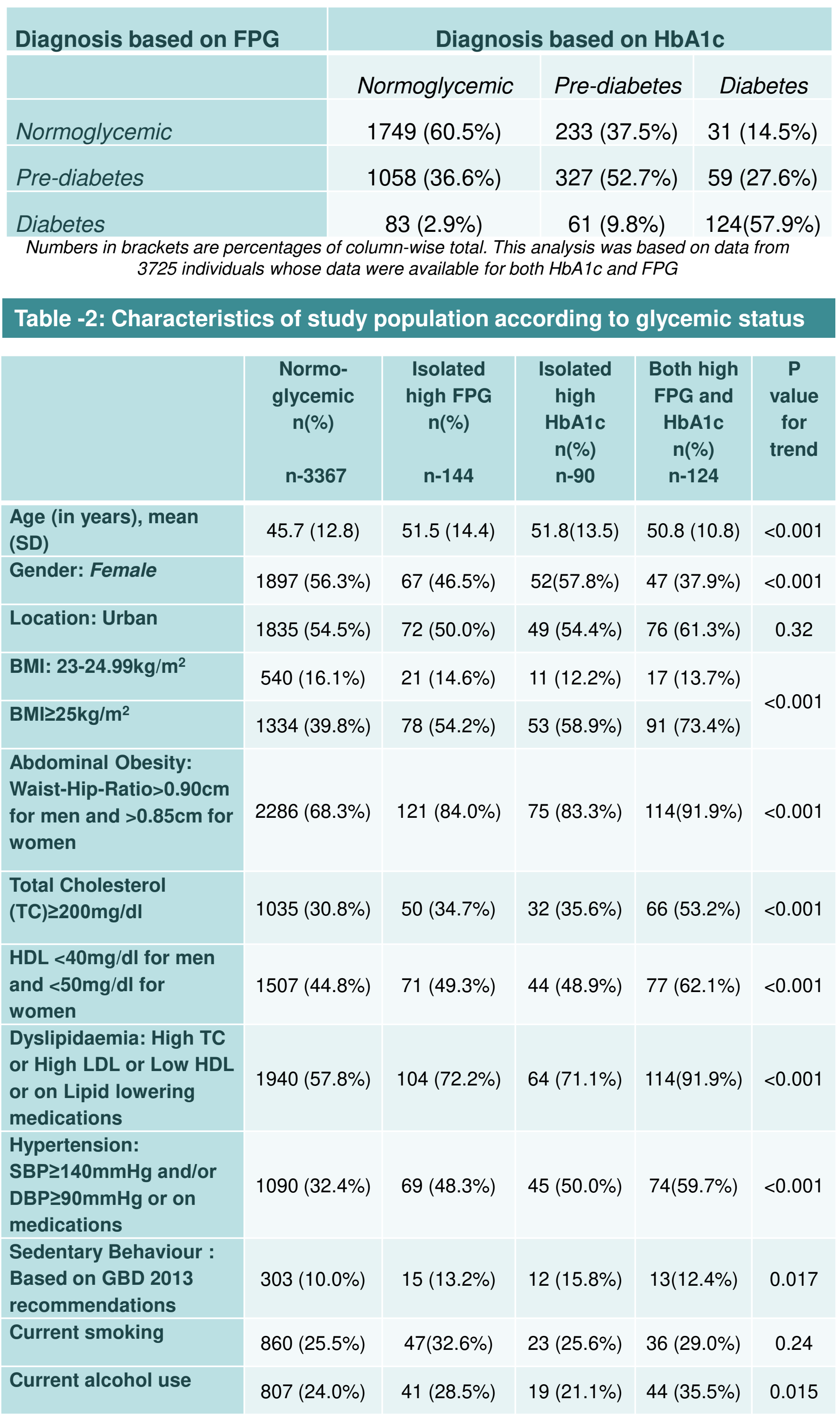

Conclusions

* The observed discordance in the diagnosis of diabetes and pre-diabetes between FPG and $\mathrm{HbA} 1 \mathrm{c}$ measurements could have significant impact on their prevalence estimation as these tests identify two different but overlapping subsets of people with hyperglycemia.

* Combined screening with FPG and HbA1c may identify individuals with high cardiovascular risk.

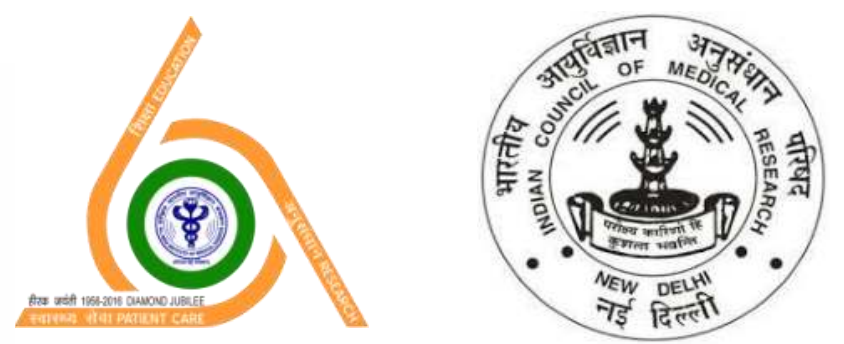

